\title{
GlueX overview: status and some future plans
}

\author{
Maria Patsyuk ${ }^{1, \star}$ on behalf of the GlueX Collaboration \\ ${ }^{1}$ Massachusetts Institute of Technology, Cambridge, MA, USA
}

\begin{abstract}
The GlueX experiment at Thomas Jefferson National Accelerator Facility (Jefferson Lab) has started data taking in late 2014 with its first commissioning beam. All of the detector systems are now performing at or near design specifications and events are being fully reconstructed. Linearly-polarized photons were successfully produced through coherent bremsstrahlung. An upgrade of the particle identification (PID) system with a GlueX DIRC detector, planned for 2018, will allow identification of final state kaons. The construction of the GlueX DIRC has already started. One of the plans for GlueX is to study properties of short-range correlations (SRC) in nuclei, which will shed new light on the quark-gluon structure of bound nucleons.
\end{abstract}

\section{Introduction}

The GlueX Experiment [1] is a key element of the Jefferson Lab $12 \mathrm{GeV}$ upgrade. The experiment is at the end of a new beamline from the Continuous Electron Beam Accelerator Facility (CEBAF) at Jefferson Lab, that will use $12 \mathrm{GeV}$ electrons to deliver linearly-polarized photons to a new experimental area, Hall D. The primary physics goal of GlueX is to discover and study the properties of hybrid mesons - particles where the gluonic field contributes directly to the $J^{P C}$ quantum numbers of the mesons [2]. Lattice QCD calculations indicate that several of the nonets of these hybrid mesons have exotic quantum numbers, forbidden $J^{P C}$ for a simple fermion-antifermion system [3]. In addition, the expected masses for the lightest hybrids are well-matched to the energy and kinematics accessible to the GlueX experiment. Commissioning of the GlueX experiment started in late 2014 and continued through the spring of 2015. The first physics quality beam is expected in Fall 2016. In this paper we present some of the first results of the GlueX experiment, as well as describe the planned upgrade of the particle identification system (PID) and plans for studying of Short Range Correlations (SRC) using photonuclear reactions and GlueX spectrometer.

\section{The Beamline}

The Hall $\mathrm{D}$ is a photon-only area taking advantage of the $12 \mathrm{GeV}$ electron beam from the upgraded CEBAF. After extraction, electrons pass through a bremsstrahlung radiator and then into the dipole magnet for tagging the energy of the scattered electrons (see figure 1 on the left). Those electrons not interacting in the bremsstrahlung radiator are deflected into an electron dump attached to the tagger hall. For the electrons producing a bremsstrahlung photon in the region between $25 \%$ and

\footnotetext{
^e-mail:mpatsyuk@mit.edu
} 
$98 \%$ of the primary electron beam energy, the scattered electrons are detected in a pair of tagger hodoscopes, thus tagging the energy of the photon. For $12 \mathrm{GeV} / \mathrm{c}$ electrons, the fine-grained Tagger Microscope (TAGM) has been placed to tag photons in the range between $8.2 \mathrm{GeV}$ and $9.2 \mathrm{GeV}$, with each microscope element spanning $10 \mathrm{MeV}$ in energy. For both higher- and lower-energy photons, the Tagger Hodoscope (TAGH) tags photons using elements about $30 \mathrm{MeV}$ wide.

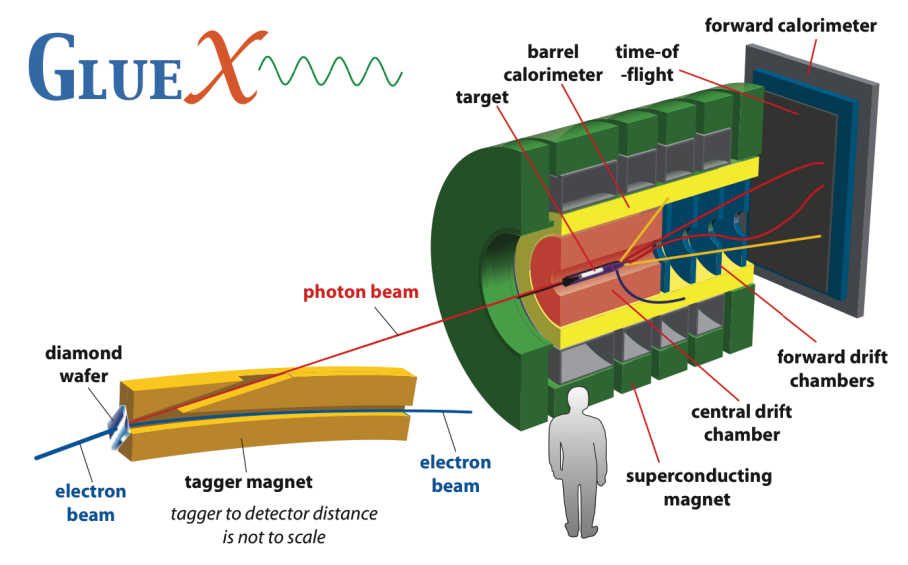

Figure 1. A schematic drawing of the photon tagger and the GlueX detector at Jefferson Lab.

To produce a linearly polarized coherent bremsstrahlung photon beam, a $20 \mu \mathrm{m}$ diamond crystal ${ }^{1}$ is utilized as a radiator. The photons in the coherent momentum range from $8.4 \mathrm{GeV} / \mathrm{c}$ to $9 \mathrm{GeV} / \mathrm{c}$ are linearly polarized relative to the crystal axes in the diamond. The peak polarization is expected to be $40 \%$. To produce two perpendicular directions of polarization, the diamond can be rotated. Coherent bremsstrahlung photons are produced in the direction of the incident electrons, while incoherent bremsstrahlung photons are produced in a cone around this direction.

The photons travel through an $80 \mathrm{~m}$ long vacuum beamline before entering Hall D. Here, the off-axis photons are removed by passing the beam through a $3.4 \mathrm{~mm}$ diameter collimator ${ }^{2}$ that has beed instrumented around the hole with an Active Collimator [4] that provides fast feedback on the beam positioning. After that photons pass through a Triplet Polarimeter that is used to monitor the linear polarization. Then photons enter a Pair Spectrometer system [6] that is used to monitor both the energy and intensity of the beam. The pair spectrometer is read out in coincidence with TAGM and TAGH, which allows determination and monitoring of the energy calibration of the tagger system. Finally photons travel into the GlueX spectrometer, where some of them interact with the liquid hydrogen target. The remainder pass into the photon beam dump at the back of Hall D.

Initial operation of the GlueX was with beam intensity of $10^{7}$ photons per second in the coherent peak (photon energy in the range $8.4-9 \mathrm{GeV}$ ), with the ultimate rate limit expected to be an order of magnitude higher.

\section{The GlueX Detector}

The GlueX detector, schematically shown in figure 1 on the right, has near uniform acceptance for both charged and neutral particles in the polar angle range from $1^{\circ}$ to $120^{\circ}$. The $30 \mathrm{~cm}$ long target

\footnotetext{
${ }^{1}$ During the spring 2015 running of GlueX at lower electron energies, $50 \mu \mathrm{m}$ and $100 \mu \mathrm{m}$ thick diamonds were used.

${ }^{2}$ During the spring 2015 run, a $5 \mathrm{~mm}$ diameter collimator was used.
} 
filled with liquid hydrogen is located in the barrel part of the GlueX detector. The solenoid magnet provides a magnetic field of about $2 \mathrm{~T}$ along the direction of the beam. Particles produced in the interaction are first coming through a scintillator-based Start Counter, which surrounds the target and the beamline in the forward direction. The light signal is detected by silicon photomultipliers $[7,8]$ providing track timing with $300 \mathrm{ps}$ resolution as well as some particle identification based on the energy loss measurement. The timing resolution is enough to identify the bucket from the electron beam $499 \mathrm{MHz}$ radio frequency (RF) structure for each photon.

The Central Drift Chamber (CDC) [9] immediately surrounding the start counter is based on straw-tube technology and provides position measurement along the charged tracks with $150 \mu \mathrm{m}$ accuracy in the $r-\phi$ plane and at the mm level along the beam axis. The CDC also provides $d E / d x$ information for charged tracks separating kaons from pions up to a momentum of $1 \mathrm{GeV} / \mathrm{c}$. The forward drift chamber (FDC) [10] complements the tracking in the forward region and performs PID by measuring $d E / d x$.

The Barrel Calorimeter (BCAL) [11-13] surrounds the tracking devices inside the solenoid. BCAL is covering the polar angle acceptance between $12^{\circ}$ and $160^{\circ}$ and reconstructs $\pi^{0}$ using decay photons of energy below $100 \mathrm{MeV}$. The energy resolution is $\sigma_{E} / E 6 \% / \sqrt{E} \oplus 2 \%$. The timing information is used to provide time-of-flight for particles interacting in the BCAL, and is used in the global PID for both charged particles and photons.

The Forward Calorimeter (FCAL) [14] is located downstream of the solenoid magnet and covers the polar angles between $1^{\circ}$ and $12^{\circ}$. FCAL is sensitive to photons with energy above $100 \mathrm{MeV}$ and has similar energy resolution.

The Time-Of-Flight (TOF) wall located upstream of FCAL consists of two layers of scintillator bars, which are read out on both sides using photomultiplier tubes. The TOF provides both timing and energy-loss information. The timing resolution of the TOF system is $100 \mathrm{ps,} \mathrm{which} \mathrm{provides} \mathrm{PID}$ for forward going particles.

All of the detector systems are now performing at or near the design specifications. Figure 2 shows an illustration of the PID capabilities for CDC, TOF, and FCAL. The left plot shows the $d E / d x$ measured by CDC for positively charged particles. Clear $\pi / p$ separation is evident up to about 1 $\mathrm{GeV} / \mathrm{c}$. In the middle plot the calculated $\beta$ of positively charged particles is shows as a function of momentum, and clear bands are seen for $\pi^{+}, K^{+}, e^{+}$, and protons, which clear proton identification up to $3 \mathrm{GeV} / \mathrm{c}$ and $\pi / K$ separation up to about $2 \mathrm{GeV} / \mathrm{c}$. The right-hand image in figure 2 shows separation of electrons/positrons from hadrons in the FCAL. The middle plot was made using the spring 2015 commissioning data, whereas the others are based on the fall 2014 commissioning data.

To fully use the potential of the GlueX detector several experiments are planned. The study of hybrid meson spectroscopy has started in 2016, the first data is sufficient to measure the beam asymmetry for a wide range of reactions $\left(\gamma p \rightarrow\left(\pi, \eta, \eta^{\prime}, \rho, \omega, \phi\right) p\right)$. The polarization transfer to the $\rho$ meson is described in detail in [18]. The physics data from 2017 and 2018 (low intensity mode with $10 \mathrm{MHz}$ in the peak) are expected to allow measurements of cross sections, spin-density matrix elements, and start of exotic hybrids search and the partial wave analysis. The measurement of the charged pion polarizability based on the Primakoff effect is planned for 2018.

The GlueX strangeness program complements the mapping of hybrid spectrum by identifying kaon final states. The quark flavor composition of a meson can be experimentally inferred by comparing the branching ratios for strange and non-strange decays. For a systematic study of the kaon final states an upgrade of the PID system is planned for 2018. A new DIRC (Detection of Internally Reflected Cherenkov light) detector [5] will be installed in the forward region of the spectrometer. After that GlueX will run in a high intensity mode ( $50 \mathrm{MHz}$ in the peak) focusing on identification of states with hidden strangeness and hyperon resonances. 

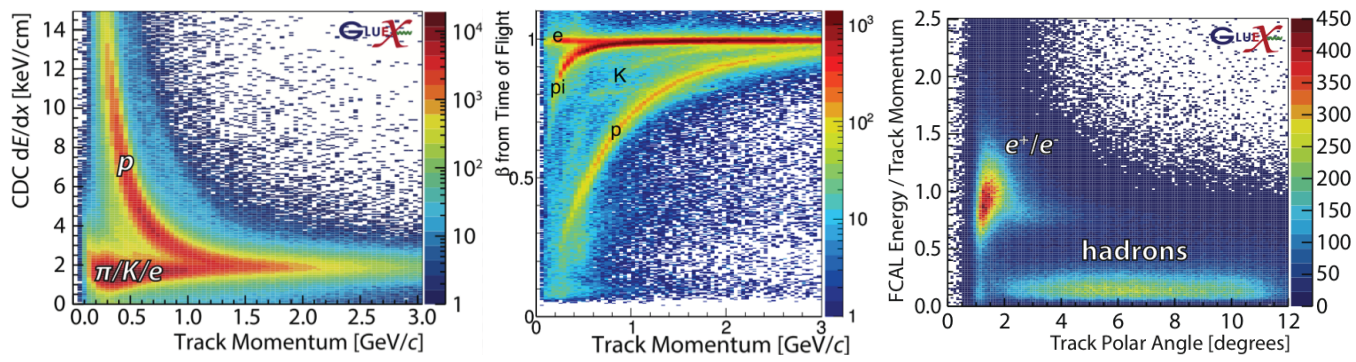

Figure 2. For positively charged particles: (Left) energy loss $d E / d x$ in the CDC as a function of reconstructed particle momentum and (Middle) the $\beta$ as determined from both fime-of-flight and path-length measurements in the detector versus the reconstructed particle momentum. The energy measured by the FCAL corresponding to the track momentum is plotted against the track polar angle on the right-hand side.

\subsection{PID Upgrade}

The proposed PID upgrade for GlueX is based on the DIRC (Detection of Internally Reflected Cherenkov light) principle [19]. The GlueX DIRC will reuse four existing BaBar DIRC [20] bar boxes filled with long and narrow radiators $\left(1.75 \times 3.5 \times 490 \mathrm{~cm}^{3}\right)$ without any modifications. Each pair of boxes will be attached to a new compact photon camera, inspired by the SuperB FDIRC prototype $^{3}$ [21]. The GlueX DIRC design is described in detail in [22]. The current GlueX DIRC design is going to provide clean separation between kaons and pions with at least three standard deviations for momenta less than $4 \mathrm{GeV} / \mathrm{c}$.

The GlueX DIRC is a thin wall of fused silica radiators located about $4 \mathrm{~m}$ away from the target, attached to photon cameras read out by an array of multi-anode photomultipliers (MaPMT). The GlueX DIRC covers the polar angle range from $2^{\circ}$ to $11^{\circ}$ and requires four unaltered BaBar bar boxes each containing 12 radiators. The bar boxes will be oriented horizontally and placed directly upstream of the TOF detector, with two bar boxes above and two below the beam. Each pair of the bar boxes will be attached to a photon camera, one of which will be located to the left, and another one to the right of the beam. The support structure of the DIRC will allow the pairs of bar boxes to slide vertically out of the active area of the detector for experiments requiring minimal material budget in front of the forward calorimeter.

The design of the photon camera is shown in figure 3. The FDIRC photon camera design is based on compact focusing blocks made of fused silica, one for each bar box in a barrel. For the GlueX planar orientation one common camera, filled with distilled water, will be used for two bar boxes together. This design with wider cameras reduces the fraction of photons reflecting on the sides, simplifying the hit pattern and removing ambiguities in the reconstruction.

Another important modification of the photon camera design is the approximation of a cylindrical mirror by three flat segments for simplicity in alignment and construction. The optimized radius of the mirror approximated by three flat segments allows using fewer photosensors, which is the main cost driver for GlueX DIRC. The resolution on the Cherenkov angle is approximately the same for the designs with the cylindrical and the three-segmented mirrors.

We plan to use H12700 [23] 64-channel MaPMTs from Hamamatsu for the read out system. The electronics design is based on the CLAS12 RICH [24], since it has similar specifications. The expected timing resolution is about $1 \mathrm{~ns}$.

\footnotetext{
${ }^{3}$ FDIRC was designed to be successor of the BaBar DIRC, but the SuperB project was canceled in 2012
} 


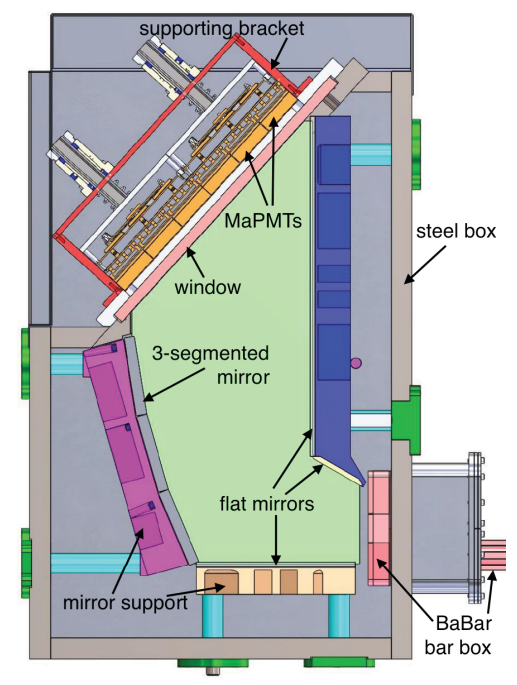

Figure 3. Schematic diagram of the cross section of the photon camera. The attached bar boxes are shown at the bottom right.

Construction has been started. The calibration system is being studied. Preparations for transporting the bar boxes from SLAC to Jefferson Lab are underway. An initial installation of two bar boxes and one photon camera in HallD is planned for 2017.

\subsection{Short Range Correlations (SRC) at GlueX}

Recent high-momentum transfer measurements [27-35] have shown that nucleons in nuclear ground states can form pairs with large relative momentum and small center-of-mass momentum of the pair because of short-range (scalar and tensor) components of the nucleon-nucleon interaction. These pairs are referred to as short-range correlated (SRC) pairs. As one form of cold dense nuclear matter fluctuations, SRC pairs are characterized by nucleonic degrees of freedom and medium densities of $\rho \approx 2-3 \rho_{0}$, where $\rho_{0}$ is the nominal density of the nucleus. Another approach to the cold dense nuclear matter fluctuations at higher densities of $\rho \approx 5-10 \rho_{0}$ involving quark degrees of freedom is being developed at JINR (Dubna) [36]. It was initially proposed by A. M. Baldin [37] in 1971.

Experimentally, SRC can be studied using a hard reaction between a high-momentum probe and a nucleon in a nucleus belonging to a SRC pair. Detection of the nucleon, its recoil partner and, if possible, the probe, sheds light on properties of SRC pairs, which define nucleon structure modifications in the nuclear medium. The current exclusive SRC studies at BNL [31-33] and JLab [35] provided statistics of tens to hundreds of events, but there are plans to perform a next generation exclusive SRC studies at GSI, JLab, and possibly, Nuclotron [38, 39], detecting tens of thousands of SRC events.

The GlueX spectrometer offers unique possibilities to measure Short Range Correlations (SRC) in exclusive photonuclear reactions using a set of thin foils instead for the liquid hydrogen target. The high energy photon beam complements the set of different probes of protons and electrons used so far and ensures hard knockout reactions. Another advantage of using a real photon is that it can interact with the neutron from a SRC pair leading to a charged final state $\left(\gamma n \rightarrow \pi^{-} p\right)$. According to the cross section of the reaction $\gamma p \rightarrow \pi^{+} n$ shown in figure 4, $\gamma p$ scattering selects mostly forward 
going high momentum proton in a nucleus. On the other hand, the GlueX detector seem to offer good neutron detection efficiency in BCAL. This allows probing $n n$ SRC pairs to understand better the role of protons versus neutrons in a nucleus.

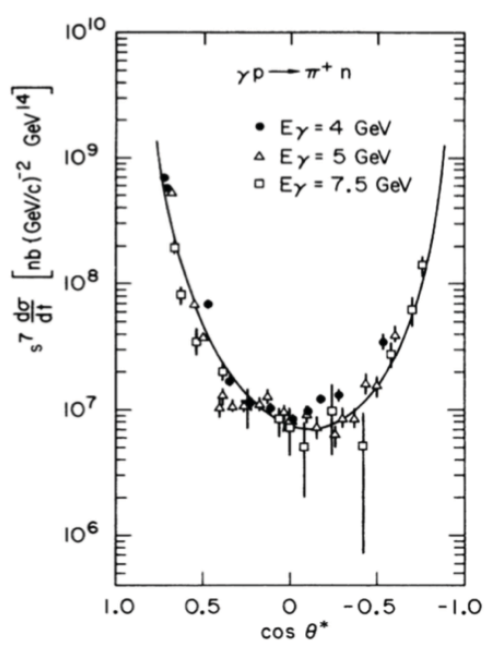

Figure 4. $s^{7} d \sigma / d t$ versus cosine of the scattering angle in the center of mass $\left(\theta^{*}\right)$. The solid line shows the empirical function $(1-z)^{-5}(1+z)^{-4}$, where $z=\cos \theta^{*}$, which is an empirical fit to the angular distribution. Figure from [40].

Thin nuclear targets will be made of ${ }^{2} \mathrm{H},{ }^{4} \mathrm{He},{ }^{12} \mathrm{C},{ }^{28} \mathrm{Si},{ }^{40} \mathrm{Ca},{ }^{48} \mathrm{Ca},{ }^{54} \mathrm{Fe},{ }^{93} \mathrm{Nb},{ }^{111} \mathrm{Ag},{ }^{208} \mathrm{~Pb}$. GlueX will measure the branching ratio for many reactions including $\gamma p \rightarrow \pi^{-} \Delta^{++}, \rho^{0} p, K^{+} \Lambda, K^{+} \Sigma^{0}$ and the equivalent reactions for neutrons.

\subsection{Summary}

The GlueX experiment has been successfully commissioned, and the first analysis based on the available data is in progress. All detector systems are near design specifications and are ready to start physics running soon. The first stage of GlueX running in $2016-2018$ at low intensity $(10 \mathrm{MHz}$ in the peak) is devoted to measurements of cross sections, asymmetries, and spin-density matrix elements, as well as identification of known resonances and hybrids using partial wave analysis.

In 2018 it is planned to increase the beam intensity by a factor of 5 to cover all parts of the GlueX exotic hybrid program and upgrade the PID system by installing a DIRC detector. This will significantly improve the separation between kaons and pions and allow effective detection of strange final states.

For a more distant future there are plans to study short range correlations in exclusive photonuclear reactions using the GlueX spectrometer.

\section{References}

[1] The GlueX Collaboration, The GlueX Experiment in Hall D, 2010, Presentation to JLab PAC 36. Available at: http://www.gluex.org/docs/pac36_update.pdf

[2] C. A. Meyer and E.S. Swanson, Prog. Part. Nucl. Phys. 82, 21-58 (2015) 
[3] J. J. Dudek et al., Phys. Rev. D 88(9), 094505 (2013). [arXiv:1309.2608 [hep-lat]]

[4] G. Miller and D. R. Walz, Nucl. Instrum. Meth. 117, 33 (1974)

[5] J. Stevens et al., JINST 11(07), C07010 (2016). [arXiv:1606.05645 [physics.ins-det]]

[6] F. Barbosa et al., Nucl. Instrum. Meth. A 795, 376 (2015)

[7] F. Barbosa, et al., Nucl. Instrum. Meth. A 695, 100 (2015)

[8] O. Soto, et al., Nucl. Instrum. Meth. A 732, 431 (2013)

[9] Y. Van Haarlem et al., Nucl. Instrum. Meth. A 622, 142 (2010). [arXiv:1004.3796 [nucl-ex]]

[10] Berdnikov, V. V. et al., Instr. Exp. Tech. 58, 25 (2015)

[11] B.D. Leverington et al., Nucl. Instrum. Meth. A 596, 327 (2008)

[12] Z. Papandreou et al., Nucl. Instrum. Meth. A 596, 338 (2008)

[13] A.E. Baulin et al., Nucl. Instrum. Meth. A 715, 48 (2013)

[14] K. Moriya et al., Nucl. Instrum. Meth. A 726, 60 (2013)

[15] GlueX Collaboration, "Mapping the spectrum of light quark Mesons and gluonic excitations with linearly polarized protons", Jefferson Lab PAC 30 Proposal (2006).

Available at: http://www.gluex.org/docs/pac30proposal.pdf

[16] A. AlekSejevs et al. [GlueX Collaboration], arXiv:1305.1523 [nucl-ex]

[17] M. Dugger et al. [GlueX Collaboration], arXiv:1408.0215 [physics.ins-det]

[18] H. Al Ghoul et al. [GlueX Collaboration], AIP Conf. Proc. 1735, 020001 (2016). [arXiv:1512.03699 [nucl-ex]]

[19] P. Coyle et al., Nucl. Inst. Meth. A 343, 292 (1994)

[20] I. Adam et al., Nucl. Inst. Meth. A 538, 281 (2005)

[21] B. Dey et al., Nucl. Instrum. Meth. A 775, 112 (2015). [arXiv:1410.0075 [physics.ins-det]]

[22] GlueX Collaboration, "GlueX DIRC Technical Design Report" (2015). Available at: http://argus.phys.uregina.argus.phys.uregina.ca/gluex/DocDB/0028/002809/003/dirc_tdr.pdf

[23] https://www.hamamatsu.com/resources/pdf/etd/H12700_TPMH1348E.pdf

[24] CLAS12 Collaboration, "CLAS12 RICH Technical Design Report" (2013). Available at: https://www.ge.infn.it/jlab12/files/RICH_TDR.pdf

[25] K. Nishimura et al., Nucl. Instr. and Meth. A 639, 177 (2011)

[26] J. Hardin and M. Williams, arXiv:1608.01180 [physics.data-an]

[27] K. S. Egiyan et al., Phys. Rev. C Nucl. Phys. 68, 014313 (2003)

[28] K. S. Egiyan et al., Phys. Rev. Lett. 96, 082501 (2006)

[29] R. A. Niyazov et al., Phys. Rev. Lett. 92, 052303 (2004)

[30] F. Benmokhtar et al., Phys. Rev. Lett. 94, 082305 (2005)

[31] J. L. S. Aclander et al., Phys. Lett. B 453, 211 (1999)

[32] A. Tang et al., Phys. Rev. Lett. 90, 042301 (2003)

[33] A. Malki et al., Phys. Rev. C Nucl. Phys. 65, 015207 (2002)

[34] E. Piasetzky et al., Phys. Rev. Lett. 97, 162504 (2006)

[35] R. Shneor et al., Phys. Rev. Lett. 99, 072501 (2007)

[36] S. S. Shimanskiy, arXiv:1411.7211 [nucl-th]

[37] A. M. Baldin, Bulletin of the Lebedev Physics Institute, LPI RAS 1, 35 (1971)

[38] B. V. Vasilishin et al., JINR Preprint 9-86-512 (Dubna, 1986)

[39] NICA Conceptual Design Report, JINR

[40] R. L. Anderson et al., Phys. Rev. D 14(3), 13 (1976) 